\title{
Motifs textuels spécifiques au genre policier et à la littérature « blanche »
}

\author{
Laetitia Gonon ${ }^{1}$, Vannina Goossens ${ }^{2}$, Olivier Kraif ${ }^{2}$, Iva Novakova $^{2}$, Julie Sorba ${ }^{2}$ \\ ${ }^{1}$ Univ. Grenoble Alpes - Litt\&Arts (UMR 5316), France \\ ${ }^{2}$ Univ. Grenoble Alpes - Lidilem (EA 609), France
}

\begin{abstract}
Résumé. L'étude propose d'analyser des motifs textuels spécifiques du roman policier et du roman de la littérature dite "blanche» de langue française. Ces motifs sont choisis selon des critères morphosyntaxiques, sémantiques, de spécificité et de dispersion : on se penchera précisément sur les motifs autour du nom de la porte pour le roman policier, et sur les motifs autour du verbe écrire pour le roman de littérature «blanche». Il s'agit de présenter leurs différentes réalisations, c'est-à-dire leur version minimale et leurs extensions syntagmatiques aussi bien que leurs variations paradigmatiques. Par la description de ces motifs, on contribue sur le plan linguistique à la réflexion sur les phénomènes de phraséologie spécifiques. Sur le plan stylistique, en s'interrogeant sur leur fonction discursive, on tente d'apporter de nouveaux éléments d'une part pour la définition des sous-genres de la paralittérature romanesque, et, d'autre part, pour la caractérisation de la langue littéraire.
\end{abstract}

\begin{abstract}
Textual patterns specific to detective novels and "white" literature. This study aims to analyze textual patterns specific to French crime and general fiction. Patterns were selected according to morphosyntactic, semantic, specificity and dispersion criteria. The focus of attention is on the patterns surrounding the noun phrase la porte (the door) in the detective novel, and patterns found alongside the verb écrire (to write) in general fiction. Consideration will be given to all the different realizations of the patterns in question, i.e. their minimal realizations, their syntagmatic extensions and their paradigmatic variations. From the point of view of linguistics, our description of these patterns seeks to make a contribution to the study of specific phraseological phenomena. From the perspective of stylistics, our exploration of the discursive functions of the patterns under survey aims to establish new criteria both for the definition of particular subgenres of popular fiction and for the characterization of literary language.
\end{abstract}

\section{Introduction}

L'étude des motifs choisis ici s'inscrit dans la continuité d'autres études pilotes menées par les membres du projet PhraseoRom, qui s'intéresse à la phraséologie étendue dans les romans contemporains, postérieurs à $1950^{1}$. Partant de l'hypothèse que la langue littéraire se caractérise par la surreprésentation statistiquement significative de lexèmes (mots-clés), de collocations ou de phraséologismes [Siepmann, 2015] qui lui sont statistiquement spécifiques, nous nous intéressons aux constructions lexico-syntaxiques (CLS) récurrentes dans certains sous-genres romanesques, afin d'identifier des motifs textuels qui leur sont propres. Nous avons ainsi déjà pu contraster des corpus français de romans policiers (POL) 
et sentimentaux (SENT) [Gonon, Goossens \& Novakova, 2018] et nous proposons d'étudier ici des motifs spécifiques à un sous-genre dit paralittéraire, le roman policier (POL) comparés à la littérature «blanche » ou générale (GEN). Notre objectif est de mettre au jour des critères aidant à l'identification et à la définition des genres romanesques, et de la langue « littéraire », en général.

Il s'agit donc d'étudier, sur le plan linguistique (syntactico-sémantique), les motifs caractéristiques de nos corpus POL et GEN de langue française, et, sur le plan stylistique, de déterminer leurs fonctions textuelles (descriptives et narratives [Adam, 2005]). Nous présenterons d'abord le cadre théorique, le corpus et la méthodologie ainsi que notre choix des motifs (partie 2). Nous analyserons ensuite les motifs autour de la porte dans le POL (partie 3) et autour d'écrire dans le GEN (partie 4).

\section{Cadre théorique, corpus et méthodologie}

Notre étude s'inspire des modèles fonctionnels et contextualistes [Sinclair, 2004], qui explorent systématiquement quatre niveaux (lexical, sémantique, syntaxique et discursif) pour l'analyse des unités linguistiques. Les motifs sont définis comme «un "cadre collocationnel" accueillant un ensemble d'éléments fixes et variables susceptibles d'accompagner la structuration textuelle, et simultanément, de caractériser des textes de genres divers » [Longrée \& Mellet, 2013: 66]. Ces motifs peuvent être également considérés comme des "unités multidimensionnelles », constituées à la fois d'associations lexicales et grammaticales, d'appariements entre forme et sens, ou entre fonction pragmatique et discursive [Legallois, 2012: 45]. Notre approche est essentiellement inductive (corpus driven), nos observations étant guidées par les données issues de vastes corpus numériques syntaxiquement annotés.

Dans ce travail, nous nous appuyons sur deux corpus de romans français contemporains: l'un composé de romans policiers $\left(\mathrm{POL}^{2}\right)$, l'autre de romans de littérature générale $\left(\mathrm{GEN}^{3}\right)$. Les caractéristiques de ces deux corpus sont présentées dans le tableau 1 ci-dessous.

Tableau 1. Composition des corpus

\begin{tabular}{|l|c|c|}
\hline & POL & GEN \\
\hline Tokens & 17859351 & 34334554 \\
\hline Auteurs & 83 & 170 \\
\hline Euvres & 194 & 445 \\
\hline
\end{tabular}

Ces corpus ont été annotés syntaxiquement au moyen de l'analyseur Xip [Aït Mokhtar, Chanod \& Roux, 2001], ce qui nous permet d'en extraire automatiquement des arbres lexico-syntaxiques récurrents (ALR) [Tutin \& Kraif, 2016]. Ces ALR regroupent des unités lexicales reliées par des dépendances syntaxiques et sont construits à partir de séries de cooccurrences statistiquement significatives (en fonction d'une mesure d'association statistique). Ils permettent de faire émerger des motifs textuels saillants. La figure 1 donne un exemple d'ALR extrait correspondant à l'expression jeter un coup d'œil à sa montre :

Fig. 1. Exemple d'arbre lexicosyntaxique récurrent (ALR)

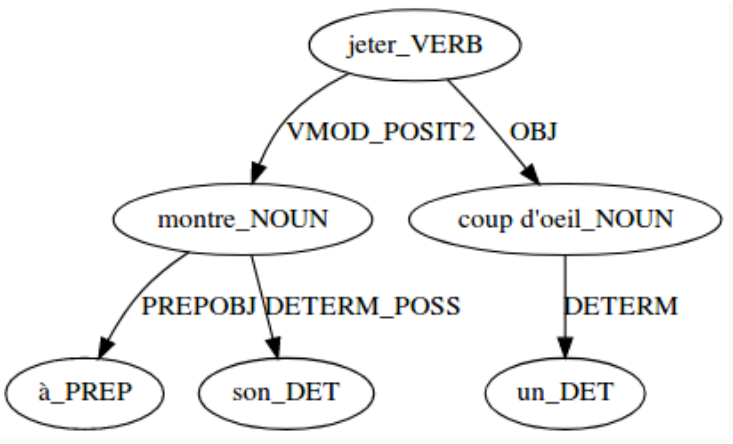


Les ALR sont extraits des deux corpus à partir des pivots nominaux et verbaux dont la fréquence est supérieure à 5, puis leurs fréquences respectives sont comparées afin de mesurer leur spécificité dans chaque corpus. Suivant la méthode Keywords [Bertels \& Speelmann, 2013], nous utilisons le calcul du rapport de vraisemblance ou log-likelihood ratio (LLR) afin de déterminer si une répartition s'écarte significativement d'une distribution aléatoire ou non. De la sorte, on peut mettre en évidence les ALR dont la fréquence relative dans l'un de nos deux corpus est significativement supérieure à la fréquence dans l'autre corpus. Les critères retenus pour la sélection des ALR représentatifs sont les suivants :

- LLR supérieur ou égal à 10,83, seuil à partir duquel la surreprésentation de l'ALR dans un corpus peut être considérée comme statistiquement significative ;

- Dispersion : ALR présent chez au moins 42 auteurs dans chacun des deux corpus (environ $50 \%$ pour le corpus POL). Le nombre d'auteurs est fixé à l'identique pour les deux corpus et permet d'isoler des ALR caractéristiques d'un sous-genre et non seulement d'un auteur ou d'une œuvre ;

- Critère morpho-syntaxique : on s'intéresse ici aux ALR contenant des verbes, pertinents pour la dimension sémantique étudiée (cf. la dimension 'action' détaillée ci-dessous). Cela permet en outre d'exclure les expressions exclusivement référentielles (ex : tous les habitants de l'immeuble, Monsieur le Procureur, les nains de jardin...).

Suite à l'application de ces critères, nous avons retenu 279 ALR spécifiques au POL et 264 ALR spécifiques au GEN. À chacun de ces ALR a été attribuée une étiquette sémantique. Le détail des dimensions et valeurs considérées, ainsi que le nombre et le pourcentage d'ALR concernés sont consignés dans le tableau 2 ci-dessous.

Tableau 2. Répartition des étiquettes sémantiques au sein des corpus

\begin{tabular}{|c|c|c|c|c|c|}
\hline \multirow{2}{*}{$\begin{array}{l}\text { Dimension } \\
\text { Action }\end{array}$} & \multirow{2}{*}{$\begin{array}{l}\text { Valeur } \\
\text { Mouvement }\end{array}$} & \multicolumn{2}{|c|}{ POL } & \multicolumn{2}{|c|}{ GEN } \\
\hline & & 42 & $15,05 \%$ & 8 & $3,03 \%$ \\
\hline & Déplacement & 27 & $9,68 \%$ & 8 & $3,03 \%$ \\
\hline & Autre & 54 & $19,35 \%$ & 37 & $14,02 \%$ \\
\hline État & & 12 & $4,30 \%$ & 15 & $5,68 \%$ \\
\hline \multirow[t]{3}{*}{ Qualia } & Affect & 3 & $1,08 \%$ & 27 & $10,23 \%$ \\
\hline & Sensation & 4 & $1,43 \%$ & 1 & $0,38 \%$ \\
\hline & Perception & 5 & $1,79 \%$ & 22 & $8,33 \%$ \\
\hline Cognition & & 31 & $11,11 \%$ & 18 & $6,82 \%$ \\
\hline \multirow[t]{2}{*}{ Setting } & Lieu & 0 & $0,00 \%$ & 3 & $1,14 \%$ \\
\hline & Temps & 9 & $3,23 \%$ & 21 & $7,95 \%$ \\
\hline \multirow[t]{2}{*}{ Caractérisation } & Objet & 0 & $0,00 \%$ & 0 & $0,00 \%$ \\
\hline & Personnage & 2 & $0,72 \%$ & 2 & $0,76 \%$ \\
\hline \multirow[t]{2}{*}{ Communication } & Physique & 9 & $3,23 \%$ & 3 & $1,14 \%$ \\
\hline & Verbale & 22 & $7,89 \%$ & 48 & $18,18 \%$ \\
\hline Autres & & 59 & $21,15 \%$ & 51 & $19,32 \%$ \\
\hline Total & & 279 & $100,00 \%$ & 264 & $100,00 \%$ \\
\hline
\end{tabular}

Nous avons choisi de nous concentrer, pour cette étude, sur des motifs appartenant à une dimension sémantique fortement représentée dans les deux corpus : les actions «autres » (en grisé dans le tableau 2), c'est-à-dire les actions qui ne concernent ni un mouvement du 
corps (secouer la tête, tendre la main...), ni un déplacement (entrer dans le bureau, quitter la pièce...). Parmi ces actions, nous avons sélectionné plusieurs ALR actualisant des motifs spécifiques au POL (les motifs de la porte) et spécifiques au GEN (les motifs de l'écriture). Les statistiques relatives à ces ALR se trouvent dans les tableaux 3 (POL) et 4 (GEN).

Tableau 3. ALR spécifiques à POL

\begin{tabular}{|l|l|l|l|l|l|}
\hline ALR & $\begin{array}{l}\text { Fréquence } \\
\text { POL }\end{array}$ & $\begin{array}{l}\text { Fréquence } \\
\text { GEN }\end{array}$ & $\begin{array}{l}\text { Dispersion } \\
\text { POL }\end{array}$ & $\begin{array}{l}\text { Dispersion } \\
\text { GEN }\end{array}$ & LLR \\
\hline $\begin{array}{l}\text { Il pousse la } \\
\text { porte }\end{array}$ & 155 & 139 & 43 & 66 & 42,18696554 \\
\hline $\begin{array}{l}\text { Il referme } \\
\text { la porte }\end{array}$ & 155 & 209 & 48 & 80 & 10,94953028 \\
\hline $\begin{array}{l}\text { Pousse la } \\
\text { porte }\end{array}$ & 339 & 408 & 62 & 109 & 39,68638229 \\
\hline $\begin{array}{l}\text { Refermer } \\
\text { la porte }\end{array}$ & 414 & 560 & 66 & 107 & 28,76771827 \\
\hline $\begin{array}{l}\text { Ouvrir la } \\
\text { porte }\end{array}$ & 825 & 1260 & 79 & 146 & 25,9363067 \\
\hline $\begin{array}{l}\text { La porte } \\
\text { s'ouvre }\end{array}$ & 500 & 570 & 71 & 119 & 71,13511914 \\
\hline
\end{tabular}

Tableau 4. ALR spécifiques à GEN

\begin{tabular}{|l|l|l|l|l|l|}
\hline ALR & $\begin{array}{l}\text { Fréquence } \\
\text { POL }\end{array}$ & $\begin{array}{l}\text { Fréquence } \\
\text { GEN }\end{array}$ & $\begin{array}{l}\text { Dispersion } \\
\text { POL }\end{array}$ & $\begin{array}{l}\text { Dispersion } \\
\text { GEN }\end{array}$ & LLR \\
\hline $\begin{array}{l}\text { Écrire un } \\
\text { roman }\end{array}$ & 26 & 123 & 11 & 42 & 20,83734122 \\
\hline Écrire lettre & 13 & 83 & 10 & 43 & 21,26784224 \\
\hline
\end{tabular}

Notons, par ailleurs, que nous présentons dans ces tableaux les versions minimalistes des ALR et que d'autres éléments peuvent venir s'intercaler entre les lemmes (p. ex. $\underline{l a}$ porte a été brusquement refermée correspond bien à l'ALR refermer la porte). Par commodité, et pour faciliter la lecture, on présente ici chaque ALR par l'une de ses réalisations canoniques.

Pour analyser ces ALR et leurs variations, nous avons également eu recours à l'étude de leur lexicogramme. Le lexicogramme établit, pour un pivot (11) et dans un corpus donné, la liste de ses cooccurrents (12) les plus fréquents en intégrant à la fois les fréquences de chaque unité (f1, f2) et celle de la cooccurrence (f), la dispersion (f. disp.) et la mesure d'association statistique (LLR). Le tableau 5 indique les 5 collocatifs verbaux les plus spécifiques du nom porte dans le corpus POL.

Tableau 5. Extrait du lexicogramme de porte dans POL

\begin{tabular}{|c|c|c|c|c|c|c|c|}
\hline 11 & 12 & $\mathrm{f}$ & f1 & $\mathrm{f} 2$ & $\mathrm{n}$ & $\begin{array}{c}\text { f. } \\
\text { disp }\end{array}$ & LLR \\
\hline porte_NOUN & ouvrir_VERB & 1893 & 58680 & 33137 & 25075570 & 80 & 8624,1326 \\
\hline porte_NOUN & refermer_VERB & 587 & 58680 & 6400 & 25075570 & 71 & 3221,2567 \\
\hline porte NOUN & fermer_VERB & 538 & 58680 & 11520 & 25075570 & 70 & 2226,541 \\
\hline porte_NOUN & frapper_VERB & 401 & 58680 & 10198 & 25075570 & 73 & 1525,2157 \\
\hline porte_NOUN & pousser_VERB & 421 & 58680 & 14284 & 25075570 & 66 & 1371,0919 \\
\hline
\end{tabular}




\section{Analyse linguistique et stylistique des motifs de la porte dans le POL}

Les motifs de la porte se déclinent sous les patrons syntaxiques suivants, qui contiennent des éléments obligatoires, constitutifs du cœur du motif (indiqués en gras), et des éléments facultatifs (placés entre parenthèses) :

$X$ pousse (violemment) la porte (vitrée) (de la salle).

$\mathrm{X}$ (lui/leur) ouvre la porte (grinçante) (de l'appartement).

$\mathrm{X}$ ferme/referme la porte (blindée) (de son bureau) (à double tour) (derrière lui).

La porte (automatique) (de la chambre) s'ouvre (brusquement).

La porte se referme (doucement) (derrière lui).

Ces configurations agrègent de nombreux éléments et présentent des variations sur le plan paradigmatique et syntagmatique. Sur le plan syntagmatique, elles apparaissent soit en version minimaliste, soit avec des extensions. Nous analyserons les distributions lexicosyntaxiques de ces motifs en les reliant systématiquement à leurs fonctions discursives. Trois cas de figure saillants se présentent pour ces motifs: en phrase indépendante, juxtaposée ou coordonnée (section 3.1) ; après des Vdicendi, de mouvement ou d'action + motif au gérondif (section 3.2) ; dans des subordonnées temporelles introduites par lorsque, dès que, avant de, après avoir, quand (section 3.3).

\subsection{Phrases indépendantes, juxtaposées ou coordonnées}

\subsubsection{Version minimaliste des motifs}

Les motifs dans leur version minimaliste apparaissent rarement en phrase indépendante, comparé à d'autres motifs ${ }^{4}: 6 \%$ pour pousser la porte; $5,3 \%$ pour refermer la porte; $3,5 \%$ pour ouvrir la porte; $2 \%$ pour fermer la porte. On les retrouve au sein de séquences mixtes (descriptives-narratives) :

(1) Au bout du chemin se dressait la chapelle des bénédictins de Ganagobie. Ils poussèrent la porte. Les moines disaient la messe. (F.-O. Giesbert, L'Immortel, 2008)

(2) Et ce colosse narquois leur tapait sur les nerfs. Il ouvrit la porte. Son sourire flottait toujours dans l'air. (J.-C. Grangé, La Forêt des mânes, 2009)

Lorsque le $\mathrm{N}$ porte est sujet ${ }^{5}$, le motif minimaliste apparaît plus souvent dans une phrase simple (20\% des cas) que lorsqu'il est c.o.d (cf. exemples de (4) à (6)), au sein de la narration :

(3) J'appuyai un léger coup sur la sonnette d'entrée. La porte s'ouvrit. Mon cœur se mit à battre. (J.-C. Izzo, Solea, 1998)

Par ailleurs, ces motifs en version minimaliste sont réalisés plus fréquemment dans des phrases juxtaposées et coordonnées. Dans cette distribution, ils font partie d'une succession d'événements au sein de séquences narratives (majoritairement au présent ou au passé simple). Ces événements sont situés dans les phrases précédant ou suivant celle du motif : 
(4) Il s'activa, les muscles douloureux, pour remplir le réservoir de la douche et celui de l'évier, puis il ferma la porte et se déshabilla. L'eau tiède lui fit du bien. (S. Brussolo, La Main froide, 1995)

(5) Il poussa la porte et s'immobilisa; une odeur de cigarette flottait dans l'air. (Ch. Brouillet, Le Collectionneur, 1995)

(6) Sans rien dire, il se leva, ouvrit la porte et fit un signe à Malko plongé dans la lecture des consignes de sécurité. (G. de Villiers, À l'ouest de Jérusalem, 1990)

Ces distributions prototypiques sont conformes à la fonction discursive (FD) des motifs dans le POL : ils constituent la charpente de la narration. En effet, ils sont, d'une part, spécifiques de l'enquête: les personnages vont d'un lieu à l'autre pour recueillir des informations (3); ou bien le coupable s'introduit chez une victime, ce qui est un topos du thriller (5), à moins que le personnage se dépêche d'aller en sauver un autre chez lui (ex. 7 ci-dessous). D'autre part, le motif entre dans une énumération de menues actions des héros dans les marges de l'intrigue (ex. 4 et 6).

Les données statistiques, issues des lexicogrammes, montrent que, dans les phrases coordonnées, ces motifs s'associent préférentiellement avec des verbes de mouvement comme entrer, sortir, pénétrer, avancer, traverser etc. Ainsi, l'association de pousser/refermer la porte avec le verbe entrer est hautement spécifique (LLR 45 pour pousser et LLR 64 pour refermer la porte). Refermer la porte attire aussi préférentiellement le verbe s'adosser (LLR 56). Ces associations statistiquement spécifiques peuvent être rapprochées des « colligations textuelles » [Hoey, 2005].

(7) Elle entra enfin chez elle, referma la porte et s'arrêta, interdite : elle trouva Harry dans la salle de bains, rasoir à la main. (B. Lenteric, Voyante, 1982)

\subsubsection{Version étendue des motifs}

Ces motifs présentent aussi différentes extensions syntagmatiques du $\mathrm{N}$ porte (sous forme d'adjectif ou de SNprép) et autour du verbe (sous forme d'adverbe ou de SNprép). Ces extensions varient aussi sur le plan paradigmatique.

\section{a) Extensions syntagmatiques du N porte (+ ADJ ou SNprép)}

Parmi les accompagnateurs les plus spécifiques du $\mathrm{N}$ porte, on retrouve, pour tous les motifs, les adjectifs vitrée, battante, blindée, grillagée, arrière, grinçante, entrouverte, cochère, etc. Les personnages poussent souvent des portes vitrées (LLR 117) :

(8) Il remonta une courte allée, sur la droite, franchit quelques marches et poussa la porte vitrée. (D. Daeninckx, Éthique en toc, 2000)

Parmi les extensions sous forme de SNprép, on trouve : de la chambre, du bureau, de l'immeuble, de la salle, de la cuisine, d'entrée, de son bureau, de la bibliothèque, de la salle de bain. Par exemple, pour ouvrir et pousser la porte, l'extension la plus spécifique est de la chambre (respectivement LLR 176 et 68) :

(9) Rhoda ferma son sac, ouvrit la porte de la chambre et s'engouffra dans le couloir.

(J. Delafosse, Le Cercle de sang, 2006)

La FD est narrative : généralement, le motif introduit une nouvelle séquence narrative (le personnage quitte un lieu, ex. 9 ; ou entre dans un nouveau lieu, ex. 8). Le motif s'accompagne souvent de qualificatifs ou de SNprép qualifiants, précisant la matière - 
vitrée, blindée -, la localisation, etc. Il entre plus largement dans une énumération d'actions exécutées par le détective (8) : c'est une manière de montrer quels espaces il traverse pour mener à bien son enquête ; la FD est donc indirectement descriptive, puisqu'elle permet de représenter l'enquêteur en nomade, toujours en mouvement.

b) Extensions syntagmatiques du verbe (+ ADV ou SNprép)

Les données montrent une préférence du motif ouvrir la porte pour les adverbes brusquement (LLR 87), brutalement (LLR 79), soudain (LLR 55), tandis que l'action de (re)fermer la porte se fait surtout sans bruit, doucement (refermer LLR 128), soigneusement (LLR 97), délicatement.

(10) Adamsberg resta immobile un instant, puis il referma doucement la porte et désigna la chaise à la jeune femme. (F. Vargas, Pars vite et reviens tard, 2001)

La FD est ici narrative : le motif entre dans une séquence prototypique du POL, comme dans l'exemple (10) qui parle de l'interrogatoire d'un témoin au commissariat (on l'écoute porte fermée).

c) Deux autres extensions spécifiques

Le motif ouvrir la porte est aussi étendu par lui/leur (LLR 110) :

(11) Brolin lui ouvrit la porte, tout habillé, le visage autant froissé que le T-shirt.

(M. Chattam, In tenebris, 2003)

Le pronom personnel datif d'intérêt (c.o.s.) introduit une personne dont on ne doute pas de l'identité : le pronom est en effet anaphorique. Cette extension est assez typique du POL : comme en (11), l'enquêteur (Brolin) est dérangé chez lui par un collègue (lui) qui a de nouvelles informations à lui communiquer (FD narrative).

En plus des éléments déjà répertoriés supra, apparait une extension de l'ensemble du motif sous forme de le SNprép : derrière lui (elle, eux).

(12) Blandine referma avec douceur la porte derrière elle. Elle n'alluma pas la lumière de peur d'être repérée par un agent congolais rôdant à tout hasard dans les jardins du Laico. (P. Besson, Mais le fleuve tuera l'homme blanc, 2009)

(13) On est venu, on l'a tué, on est reparti, en refermant soigneusement la porte derrière soi. (M. G. Dantec, Metacortex, 2010)

Ainsi, les motifs autour de fermer/refermer la porte s'avèrent les plus « fournis » sur le plan linguistique, donc les plus «polylexicaux», car ils agrègent le plus grand nombre d'éléments autour de leur cœur.

Le motif peut alors avoir une fonction double : sur le plan narratif d'une part, il clôt ou ouvre une séquence - en (13), l'assassin désigné par on disparait après avoir refermé la porte, il a fini de commettre son crime. La seconde fonction du motif est donc parfois descriptive pour créer une atmosphère : refermer la porte induit souvent un effet de suspense. Ainsi en (12), Blandine a crocheté une serrure et a pénétré, en pleine obscurité, dans une chambre d'hôtel qu'elle veut fouiller sans attirer l'attention. 


\subsection{Vdicendi, de mouvement ou d'action + motif au gérondif}

Souvent, lorsqu'ils se combinent avec des verbes dicendi, de mouvement ou d'action, les motifs, dans leur version minimaliste ou étendue, apparaissent au gérondif. L'action de pousser, ouvrir, fermer la porte est alors concomitante à d'autres actions liées aux déplacements des personnages ou à leurs actes de parole. Cette distribution est très spécifique pour refermer la porte (LLR 122) et ouvrir la porte (LLR 98) et un peu moins pour pousser la porte :

(14) Celui-ci quitta aussitôt le bureau en fermant précautionneusement la porte.

(Th. Jonquet, Le Manoir des immortelles, 1986)

(15) - Vous avez été longs! s'écria Emma en ouvrant la porte. J'imaginais déjà le pire! (F. Thilliez, La Forêt des ombres, 2006)

Ici la FD du motif est une fois de plus narrative : la fermeture ou l'ouverture de la porte permet d'embrayer sur une nouvelle séquence, puisque le nombre de personnages présents change. En (14) par exemple, le personnage est laissé seul dans le bureau pour réfléchir; en (15), les deux personnages accueillis par Emma retrouvent le confort de l'intérieur après un tour sous la neige. Avec le gérondif, ce motif est moins caractéristique de l'intrigue du POL : il reste à l'arrière-plan de l'enquête (il accompagne seulement une autre action, quitta le bureau, s'écria).

\subsection{Dans des subordonnées temporelles introduites par quand, lorsque, dès que, avant, après}

Nos motifs autour de la porte se retrouvent aussi dans des subordonnées temporelles introduites par lorsque, quand, dès que, avant, après. Par exemple, le motif ouvrir la porte attire préférentiellement quand (LLR 69), lorsque (LLR 65), tandis que refermer la porte s'associe davantage avec dès que (LLR 98). Par ailleurs, ces subordonnées ont une préférence pour la position initiale de la phrase («colligations textuelles », Hoey 2005) : elles donnent un cadre à l'action (valeur temporelle des passés antérieurs, aspect tensif du gérondif). Elles renvoient à des actions aspectuellement brèves qui ponctuent les moments charnières de la narration :

(16) Lorsqu'elle ouvrit la porte, elle eut la surprise de tomber sur un homme d'une cinquantaine d'années, maigre, au visage long et las, qui la salua sans sourire mais poliment. (P. Senécal, Les Sept Jours du talion, 2002)

(17) Quand Nicolas eut poussé la porte, il fut frappé par les inconvénients intérieurs de la situation de la boutique. (J.-F. Parot, L'Énigme des blancs-manteaux, 2000)

(18) Dès qu'elle eut refermé la porte, Charlotte passa dans son bureau et se connecta à Internet. (A. H. Japp, Les Cadavres n'ont pas froid aux yeux, 2012)

Le motif au gérondif a aussi une préférence pour le début de phrase où il apparait en apposition (« colligation textuelle ») :

(19) En ouvrant la porte d'entrée de la maison, Harry ne masqua pas son étonnement. (J. Dicker, La Vérité sur l'affaire Harry Quebert, 2012)

La FD est, une fois de plus, narrative : dans les différents exemples, le motif dans la subordonnée permet de passer à une autre séquence narrative, parallèlement à un changement de lieu du personnage. Souvent, c'est aussi une manière d'embrayer sur une 
séquence descriptive évoquant les affects du personnage qui découvre ce qu'il y a derrière la porte : son étonnement (19), la surprise (16), fut frappé par (17).

\section{Analyse linguistique et stylistique des motifs autour du verbe écrire dans le GEN}

Dans la littérature «blanche» ou générale, le verbe écrire apparait dans deux ALR spécifiques : écrire une lettre et écrire un roman. Si l'on observe la combinatoire du verbe écrire dans le GEN, on dénombre 93 collocatifs nominaux différents (lettre, livre, roman, poème, ligne, article, page, scénario, etc.) parmi lesquels lettre occupe le premier rang (LLR 2038) et roman le troisième (LLR 763). Notre étude se fonde sur l'analyse des 250 occurrences du motif écrire_DET_lettre et des 137 occurrences du motif écrire_DET_roman. Ces deux motifs ont en commun une même distribution du déterminant. En effet, même si le déterminant défini ou possessif n'est pas inconnu dans leur réalisation syntaxique, tous deux privilégient fortement la construction avec déterminant indéfini singulier $(67 \%$ pour écrire_DET_roman et $68 \%$ pour écrire_DET_lettre). Par ailleurs, ils présentent tous deux une version minimaliste autour de laquelle peuvent s'agréger de nombreux éléments traduisant des variations paradigmatiques et syntagmatiques. Nous analyserons ces variations en les reliant systématiquement à leurs fonctions discursives. Nous présenterons d'abord la version minimaliste de ces deux motifs (section 4.1), puis leurs versions étendues (section 4.2), et, enfin, la variété des constructions verbales dans lesquelles ils entrent (section 4.3).

\subsection{Version minimaliste des motifs}

Les deux motifs peuvent apparaitre dans leur version minimaliste $(\mathrm{V}+\mathrm{DET}+$ roman/lettre, cf. ex. 20 et 21). Toutefois, cette réalisation syntaxique est privilégiée par écrire_DET_roman (par ex., on trouve 35\% de la version minimaliste en finale absolue de la phrase au regard de $18 \%$ pour écrire_DET_lettre).

(20) Henri aime écrire des lettres. (F. Seyvos, Le Garçon inclassable, 2013)

(21) - J'écris un roman. (Ph. Sollers, Femmes, 1983)

Il n'est pas anodin que ces deux motifs soient spécifiques du GEN : le romancier projette ainsi son travail d'écriture dans l'univers de fiction. Les activités scripturales, littéraires (roman) ou intimes (lettre), manifestent sans doute l'attention que l'auteur porte au travail de la langue.

\subsection{Version étendue des motifs}

Sur le plan paradigmatique, écrire_DET_roman privilégie les variations en contexte droit (38\%) plutôt qu'en contexte gauche (4\%) autour du pivot roman :

$\mathbf{X}$ écrit DET (bon, tout, nouveau, grand, premier) roman (nazi, historique, sur la collaboration, dans lequel les révolutionnaires mexicains pourchassent et fusillent les curés, formidables, en deux jours, à succès, etc.).

En contexte gauche n'apparaissent que des modifieurs adjectivaux du nom roman tandis qu'en contexte droit sont aussi utilisés des modifieurs de toute la collocation (en deux jours, en cachette, etc.). 
En contraste, le motif écrire_DET_lettre est accompagné beaucoup plus souvent de variations dans les contextes droit (70\%) et gauche (42\%) autour du pivot lettre :

$\mathbf{X}$ écrire (à son amant, chaque après-midi, en secret, etc.) DET (longue, fichues, etc.) lettre (indignée, au maire, de sa grande écriture nerveuse, par semaine, en langue française, à sa mère, d'amour, etc.).

Le destinataire de la lettre peut apparaitre soit à l'intérieur de la collocation entre le $\mathrm{V}$ et le $\mathrm{N}$ ( $X$ écrit à son amant une lettre), soit comme extension syntagmatique de la collocation ( $X$ écrire une lettre à sa mère).

En comparant les modifieurs qui affectent les deux motifs, on s'aperçoit que tous deux partagent des extensions syntagmatiques aux valeurs semblables pour indiquer la thématique de l'écrit (écrire un roman d'espionnage, écrire une lettre d'amour), les modalités de l'écriture (écrire un roman en deux jours/en cachette ; écrire une lettre de sa grande écriture nerveuselen secret) ou un jugement axiologique (écrire un roman très compliqué, écrire un bon roman ; écrire ses fichues lettres).

Néanmoins, écrire_DET_lettre se distingue de écrire_DET_roman car il est le seul à accepter une extension syntagmatique $\grave{a} S N$ qui indique le destinataire dans $X$ écrit une lettre à $Y$ (à sa mère, à sa sæur, à son amant, au maire, etc.). Pour sa part, écrire_DET_roman se distingue du précédent par des extensions syntagmatiques indiquant un titre d'œuvre (écrire son roman intitulé Les Faux Monnayeurs/qui s'intitulait Hygiène de l'assassin) ou les modalités de la réception (écrire un roman à succès).

L'ensemble de ces observations permet de conclure que le motif écrire_DET_roman apparait plus souvent dans sa version dépouillée minimaliste que écrire_DE $T$ _lettre. On peut noter d'ores et déjà qu'avec le $\mathrm{N}$ roman, le motif est plus rigide, puisqu'il offre moins de variations qu'avec le $\mathrm{N}$ lettre. Les variations les plus intéressantes pour ces deux motifs concernent les constructions verbales : nous analyserons donc plus en détail les variations paradigmatiques pour écrire.

\subsection{Variation des constructions verbales}

\subsubsection{Les temps verbaux}

Les deux ALR hautement spécifiques extraits écrire une lettre / écrire un roman pointent une préférence marquée pour un temps verbal : le passé simple pour écrire_DET_lettre (22) et le présent pour écrire_DET_roman (23). L'étude des occurrences confirme ce point comme le montre le tableau 6 ci-dessous :

Tableau 6 : Répartition des temps verbaux du présent et du passé pour les 2 motifs

\begin{tabular}{|l|l|l|l|l|}
\hline & présent & passé simple & passé composé & imparfait \\
\hline écrire_DET_lettre & 25 occ. $(10 \%)$ & 46 occ. $(18 \%)$ & 24 occ. $(10 \%)$ & 29 occ. $(12 \%)$ \\
\hline écrire_DET_roman & 26 occ. $(19 \%)$ & 3 occ. $(2 \%)$ & 5 occ. $(4 \%)$ & 6 occ. $(4 \%)$ \\
\hline
\end{tabular}

(22) Ce fut l'anniversaire de Woglinde, je lui écrivis une lettre où je me servis de toutes mes encres et je lui envoyai une boîte de calissons d'Aix et deux albums de trois kilos chacun, Tous les animaux $d u$ monde, avec des photos pleine page de poissons ahurissants, elle qui aimait tant que je l'emmène à l'aquarium du musée de la Porte Dorée, où elle reprochait aux crocodiles d'être toujours en train de dormir. (F. Weyergans, Trois jours chez ma mère, 2005)

(23) Elle avait dit: "Ah bon, tu écris un roman ?»(D. Foenkinos, Les Souvenirs, 2011) 
En (22), la FD semble au premier abord narrative: le motif apparait dans une énumération de propositions, le narrateur expliquant ainsi l'emploi de son temps chez sa mère. Cependant, elle est surtout descriptive: le narrateur se représente ainsi comme écrivant, en attendant de (re)devenir écrivain ${ }^{6}$.

Le motif écrire un roman privilégie le présent de l'indicatif (temps de l'énonciation), puisqu'il apparait très souvent dans le discours direct, comme en (21) et (23) : dans ce dernier exemple, le narrateur fait connaissance avec une jeune fille, et se définit donc auprès d'elle comme un écrivain (FD descriptive).

L'emploi du verbe écrire à un temps conjugué est plus dominant dans le motif écrire_DET_lettre $(65 \%)$ que dans écrire_DET_roman (42\%) car ce dernier apparait davantage sous la forme infinitive ( $52 \%$ vs $\overline{3} 1 \%$ ), étudiée dans la section suivante.

\subsubsection{Les constructions à l'infinitif}

Une autre variation des motifs se réalise dans des constructions à l'infinitif. Lorsque cellesci se trouvent en position syntaxique de sujet $(24,26)$ ou d'attribut $(25)$, elles ne sont employées que marginalement pour écrire_DET_roman (5\%) et écrire_DET_lettre $(7 \%)$ :

(24) Écrire un roman accaparait. (Ph. Djian, Incontrôlables, 2009)

(25) Mon rêve depuis toujours est d'écrire des romans comiques, alors pourquoi faut-il que j'aligne des personnages semi-tarés, emportés au loin par l'ivresse de la destruction? (F. Humbert, L'Origine de la violence, 2009)

(26) Écrire une lettre à quelqu'un, c'est vouloir attirer son attention (A. Nothomb, Une forme de vie, 2010)

Ainsi, la construction avec être attributif induit une FD de commentaire, puisqu'elle permet une réflexion du narrateur, soit sur lui-même (25), soit en termes généraux (ex. 24 et 26) : le motif a une dimension réflexive (un écrivain parle du processus d'écrire).

L'infinitif complément est l'emploi principal de la forme infinitive pour les deux motifs écrire_DET_roman (44\%) et écrire_DET_lettre (27\%). Notons qu'il existe une plus grande variété de locutions verbales introductrices pour écrire_DET_lettre (20 verbes/locutions verbales différentes pour écrire_DET_roman vs 36 pour écrire DET_lettre). Et pour les deux motifs, la forme infinitive complète majoritairement un verbe ou une locution verbale ${ }^{7}(72 \%$ pour roman et $95 \%$ pour lettre) :

$\mathbf{X}$ venir de, être en train de, décider de, devoir, tenter de, envisager de, pouvoir, commencer à, vouloir écrire_DET_roman.

$\mathbf{X}$ être en train de, commencer à, demander de, vouloir, avoir la manie de, aider à, pouvoir, parvenir à, entreprendre de, se mettre à, prendre la liberté de, avoir l'audace $d$ 'écrire_DET_lettre.

En voici deux exemples issus du corpus GEN :

(27) Bien sûr on aurait pu envisager d'écrire un roman proustien jet set [...] ; ça n'aurait eu aucun intérêt (M. Houellebecq, Les Particules élémentaires, 1995)

(28) Rose aussi était en train d'écrire une lettre : en réponse à une autre dont Aline a $\mathrm{pu}$ la veille reconstituer l'enveloppe mise en petits morceaux dans la corbeille (H. Bazin, Madame Ex, 1975) 
La FD en (27) reste de commentaire : le narrateur juge des possibilités narratives, les distinctions opérées par Proust n'ayant selon lui plus aucun intérêt aujourd'hui. En (28), la FD est narrative : le groupe verbal montre l'action en déroulement.

C'est bien cette configuration syntaxique - le groupe infinitif dans un emploi de dépendance - qui est la plus spécifique de ces motifs du GEN : écrire est soumis à différents affects, souhaits, ou temporalités.

\subsubsection{Les variations aspectuelles et les modalités}

L'emploi fréquent de la forme infinitive révèle une autre spécificité de ces motifs : leur grande variation aspectuelle. Le procès peut être saisi à son début : commencer à, décider de, tenter de, se préparer à écrire_DET_roman; commencer à, entreprendre de, se mettre à, essayer de, se proposer de écrire_DET_lettre.

(29) Je pense que c'est sans doute ce soir-là que Diodème a commencé à m'écrire sa lettre. (Ph. Claudel, Le Rapport de Brodeck, 2007)

(30) Une nuit, j'ai rêvé que je me préparais à écrire le roman d'Horace : je me demandais même comment j'avais pu ne pas y penser plus tôt. (A. Maillet, Chronique d'une sorcière de vent, 1999)

Ici le motif intervient dans les marges de l'action, et participe d'une fonction cognitive en (29) - il intervient dans une subordonnée exprimant l'objet de la réflexion du narrateur. En (30), le motif intervient également dans un passage introspectif; il a une FD davantage narrative, puisque ce rêve pousse le personnage vers un projet d'écriture.

Le procès est également représenté en cours de déroulement: être en train de, écrire_DET_roman/lettre; ou à sa fin : venir de, écrire_DET_roman; parvenir à, venir de écrire_DET_lettre. Le procès peut être itératif ou continuatif : se remettre à, passer son temps à écrire_DET_roman ; avoir la manie de, passer deux heures à écrire_DET_lettre. Et il peut intervenir dans une périphrase factitive (causative) dans le cas de écrire_DET_lettre :

(31) Quand il s'agissait de lui faire écrire une lettre à un juge ou à un ministre, on ne se privait pas. (R. Ducharme, L'Avalée des avalés, 1966)

(32) Mais on a pu faire écrire la lettre par quelqu'un d'autre. (M. Laberge, Revenir de loin, 2010)

En (31), la FD est narrative : en microcontexte, l'écriture de la lettre entraine une conséquence sur le plan chronologique. Mais en macrocontexte, la FD du motif est en réalité descriptive : le locuteur accuse son épouse de l'avoir poussé à écrire à des personnes influentes pour protéger ses frères. Le motif entre donc dans un portrait à charge de l'allocutaire. En (32), la FD dépend de même de ce qui précède : ici elle est cognitive ou de commentaire, les personnages s'interrogeant sur des circonstances de l'intrigue.

Enfin, le motif à l'infinitif se retrouve dans des locutions verbales exprimant les modalités du procès :

- la volition: vouloir, décider de, promettre de, écrire_DET_roman; vouloir, prendre la liberté de, prendre la peine de, écrire_DET_lettre

- la potentialité : pouvoir écrire_DET_roman

- l'obligation : falloir écrire_DET_roman; falloir, être contraint de, obliger à écrire_DET_lettre 
(33) Écrire est la seule fenêtre, et «il faudrait écrire des romans du matin au soir ».

(Ph. Sollers, Les Voyageurs du temps, 2009)

(34) Selon lui, c'était très intéressant de vouloir écrire des romans comme j'en avais l'intention mais il était plus prudent d'obtenir quelques «diplômes » (P. Modiano, Un cirque passe, 1992)

Dans ces cas, la FD du motif participe du commentaire des locuteurs : le romancier Céline dans le premier cas (33), le narrateur de Sollers prenant des notes sur ses écrits ; le père du narrateur dans le second (34).

Enfin, le gérondif est seul attesté et de manière anecdotique pour écrire_DET_lettre (2 occ.), alors qu'il est beaucoup plus fréquent pour le motif de la porte. Cette tendance corrobore nos observations selon lesquelles les motifs autour d'écrire ont une FD beaucoup moins narrative.

\section{Conclusion}

Notre objectif était de mettre au jour des critères aidant à la définition des genres romanesques et de la langue "littéraire », en général, grâce à l'identification de motifs textuels. Notre étude linguistique et stylistique révèle que les motifs spécifiques à POL (les motifs autour de la porte) et les motifs spécifiques à GEN (les motifs autour de l'écriture) ont à la fois des configurations lexico-syntaxiques différentes et des fonctions discursives variées. Par exemple, alors que les motifs de la porte se rencontrent fréquemment avec un verbe au gérondif ou à l'intérieur d'une subordonnée temporelle, ce n'est pas du tout le cas des motifs autour de l'écriture qui privilégient, de leur côté, les constructions à l'infinitif et une grande variation aspectuelle.

Ces particularités linguistiques révèlent des fonctions discursives différentes et plus ou moins variées. En effet, dans le corpus POL, la FD des motifs autour de la porte est majoritairement narrative puisque ces derniers contribuent au développement de l'intrigue policière ; ils constituent la charpente de la narration en ouvrant ou clôturant une séquence. Néanmoins, la FD narrative peut parfois être indirectement descriptive quand les motifs peignent le personnage en détective ou créer une atmosphère particulière.

Notre étude révèle un fait particulièrement remarquable dans le corpus GEN : les seules FD narrative et descriptive, même si elles rendent compte d'un grand nombre de motifs, ne suffisent pas à les commenter tous. La plus grande complexité syntaxique du texte littéraire et une dimension métalittéraire caractéristique du corpus nous engagent alors à proposer des FD cognitive et de commentaire pour rendre compte du processus d'écriture et de la dimension réflexive mise en œuvre par l'auteur dans ce corpus GEN.

Ainsi, les motifs textuels que nous avons identifiés sur la base des constructions lexicosyntaxiques (CLS) récurrentes dans les deux corpus fournissent des critères pertinents pour contraster les genres romanesques et faire ressortir des spécificités de la langue littéraire. Nous poursuivrons dans la même perspective l'étude des spécificités phraséologiques des sous-genres romanesques en contrastant d'autres corpus, comme HIST et GEN, sciencefiction et fantasy: les critères de définition de ces catégories, que l'on établit parfois intuitivement ou de façon très générale, pourront trouver des données statistiques et des analyses précises auxquelles s'adosser. 


\section{Références bibliographiques}

Adam, J.-M. (2005). Les Textes : types et prototypes. Récit, description, argumentation, explication et dialogue. Paris : Armand Colin.

Ait Mokhtar, S., Chanod, J.-P. \& Roux, C. (2001). Robustness beyond Shallowness: Incremental Deep Parsing. Natural Language Engineering, 8, 121-144.

Bertels, A. \& Speelmann, D. (2013). 'Keywords Method' versus 'Calcul des Spécificités'. A comparison of tools and methods. International Journal of Corpus Linguistics, 18.4, 536-560.

Gonon, L., Goossens, V. \& Novakova, I. (2018, sous presse). Les phraséologismes spécifiques à deux sous-genres de la paralittérature : le roman sentimental et le roman policier. Actes du colloque Phraséologie française. Paris : Hermann.

Hoey, M. (2005). Lexical priming: a new theory of words and language. Londres/New York : Routledge.

Legallois, D. (2012). La colligation : autre nom de la collocation grammaticale ou autre logique de la relation mutuelle entre syntaxe et sémantique ?. Corpus, 11, 31-54.

Longrée, D. \& Mellet S. (2013). Le motif: une unité phraséologique englobante ? Étendre le champ de la phraséologie de la langue au discours. Langages, 189, 68-80.

Siepmann, D. (2015). A corpus-based investigation into key words and key patterns in post-war fiction. Functions of Language, 22.3, 362-399.

Sinclair, J. (2004). Trust the Text: Language, Corpus and Discourse. Londres : Routledge.

Tutin, A. \& Kraif, O. (2016). Routines sémantico-rhétoriques dans l'écrit scientifique de sciences humaines : l'apport des arbres lexico-syntaxiques récurrents. Lidil, 53, 119-141.

\footnotetext{
1 Projet ANR-DFG 2016-2020, dirigé par Iva Novakova (Université Grenoble Alpes) et Dirk Siepmann (Université d'Osnabrück), https://phraseorom.univ-grenoble-alpes.fr/accueil

${ }^{2}$ Euvres de Jean-Christophe Grangé, Maxime Chattam, Andrea H. Japp, etc. On s'est fondé, pour la constitution de ce corpus POL, sur la notoriété des auteurs, et leur appartenance incontestable au genre du roman policier.

${ }^{3}$ Euvres de Amélie Nothomb, David Foenkinos, Jean Echenoz, etc. Pour la constitution de ce corpus GEN, on s'est fondé sur la notoriété «littéraire» ou reconnue telle par l'instituion (université, prix littéraires, critiques littéraires, édition). On a donc parfois dû se livrer à des choix: certains textes de Jean Echenoz tirent vers le roman policier, mais sont publiés par des maisons généralistes, et pas dans des collections particulières.

${ }^{4}$ Par ex. ceux des menus comportements quotidiens dans le POL et le SENT.

${ }^{5}$ Cette variation du motif avec le $\mathrm{N}$ porte comme sujet (La porte s'ouvre, se referme) présente les mêmes distributions syntaxiques et les mêmes caractéristiques linguistiques et stylistiques que lorsque le $\mathrm{N}$ est c.o.d ; (ouvrir, refermer la porte). C'est la raison pour laquelle nous n'allons pas l'analyser de manière systématique.

${ }^{6}$ Le passage qui précède notre citation est en effet celui-ci : « je comparais la rédaction de ces lettres aux exercices que font les danseurs à la barre afin de s'assouplir avant le spectacle. Ces lettres me maintenaient en forme et me permettraient de passer un jour à l'essentiel, à ce que mon contrat appelait "le prochain roman de l'auteur". »

${ }^{7}$ Très rarement, on trouve aussi l'infinitif dépendant d'un nom : le désir, l'idée de, l'envie de, la difficulté de, la question de écrire_DET_roman ; l'envie de, l'idée de, le temps de écrire_DET_lettre.
} 